\title{
Trombose de Stent Farmacológico no "Mundo-Real": Análise Crítica do Registro DESIRE (Drug-Eluting Stent in the Real World)
}

\author{
Ricardo A. Costa ${ }^{1}$, Amanda G. M. R. Sousa ${ }^{1}$, Adriana Moreira ${ }^{1}$, José Ribamar Costa Jr. ${ }^{1}$, \\ Galo Maldonado', Manuel Cano', Luiz F. Tanajura', Fausto Feres' ${ }^{1}$, Luiz A. Mattos', \\ Rodolfo Staico ${ }^{1}$, Alexandre Abizaid', César Jardim¹, leda M. Liguori', \\ Abrão Cury ${ }^{1}$, Otávio Berwanger ${ }^{1}$, Adib D. Jatene' ${ }^{1}$, J. Eduardo Sousa ${ }^{1}$
}

\section{RESUMO}

Fundamentos: Reportamos a incidência e os preditores de trombose de stent numa grande coorte de pacientes complexos tratados com stents farmacológicos (SF) no "mundo-real". Método: Entre maio de 2002 e janeiro de 2008, 2.365 pacientes não-selecionados com $\geq 1$ lesão coronária com estenose $\geq 50 \%$ foram tratados com SF. O seguimento clínico até cinco anos (média 2,2 $\pm 1,5$ anos) foi completado em 98,3\%. Resultados: Entre os pacientes avaliados, 29\% tinham diabetes, $60 \%$ eram portadores de doença multiarterial e $40 \%$ apresentavam síndrome coronária aguda (15\%, infarto agudo do miocárdio [IAM]). A artéria descendente anterior foi o vaso-alvo mais freqüentemente tratado $(35,8 \%)$, e $67 \%$ das lesões eram de alta complexidade (tipo B2/C). No total, 3.634 SF foram implantados e $40 \%$ dos pacientes receberam múltiplos stents. A trombose de stent (classificação Academic Research Consortium) incidiu em 1,6\% ( $\mathrm{n}=38), 60,5 \%$ com confirmação angiográfica, $42 \%$ ocorreram entre 1 e 12 meses e $47 \%$ dos eventos tiveram desfecho fatal. Os preditores independentes de trombose de stent foram: tabagismo atual (razão de risco [RR] 2,59; intervalo de confiança [IC] 95\% 1,18-5,67; p = 0,018), intervenção coronária percutânea (ICP) no IAM (RR 3,50; IC 95\% 1,31-9,40; $p=0,013$ ), calcificação moderada/grave (RR 2,38; IC 95\% $1,34-4,23 ; p=0,003$ ), lesão excêntrica (RR 1,86; IC $95 \%$ $1,03-3,34 ; p=0,039)$, $>1$ SF implantado por território miocárdico (RR 1,81; IC 95\% 1,09-3,02; $p=0,023$ ), pósdilatação (RR 0,50; IC 95\% 0,29-0,90; p = 0,020) e estenose residual intra-stent (RR 1,04 [por aumento de unidade \%]; IC 95\% 1,01-1,06; $p=0,003)$. Conclusões: Nesse registro prospectivo do "mundo-real", a incidência cumulativa de trombose de stent até cinco anos de seguimento foi rara $(1,6 \%)$, e foi associada a tabagismo, ICP no IAM, morfologia

\section{SUMMARY}

Stent Thrombosis After Drug-Eluting Stenting in the "Real-World": A Critical Analysis of the DESIRE (Drug-Eluting Stent in the Real World) Registry

Background: We report the incidence of stent thrombosis (ST) predictors in a large cohort of complex patients treated with drug eluting stents (DES) in the real world. Methods: From May 2002 until January 2008, 2,365 non-selected patients with $\geq 1$ coronary lesion with stenosis of $\geq 50 \%$ were treated with DES. The clinical follow-up up to five years (mean time $2.2 \pm 1.5$ years) was completed in $98.3 \%$. Results: Twenty nine percent of patients had diabetes and $60 \%$ multi-vessel disease, $40 \%$ presented acute coronary syndrome $(15 \%$, acute myocardial infarction [AMI]). The anterior descending artery was the target vessel most frequently treated $(35.8 \%)$, and $67 \%$ were complex lesions (Type B2/C). In all, 3,634 DES were implanted and 40\% received multiple stents. Stent thrombosis (classification of the Academic Research Consortium) occurred in $1.6 \%(\mathrm{n}=38)$, $60.5 \%$ with angiographic confirmation, $42 \%$ occurred between 1 and 12 months and $47 \%$ of the events had a fatal outcome. The independent predictors of ST were: current smoking [relative risk (RR) 2.59; 95\% confidence interval (CI) 1.18-5.67; $\mathrm{p}=0.018]$, AMI intervention (RR 3.50; 95\% Cl 1.31-9.40; $\mathrm{p}=0.013)$, moderate to severe calcification (RR 2.38; 95\% Cl 1.34-4.23; $p=0.003$ ), excentric lesion ( $R R$ $1.86 ; 95 \% \mathrm{Cl} 1.03-3.34 ; \mathrm{p}=0.039),>1$ DES implanted per myocardial territory (RR 1.81; 95\% Cl 1.09-3.02; $\mathrm{p}=0.023)$, post-dilatation ( $\mathrm{RR} 0.50 ; 95 \% \mathrm{Cl} 0.29-0.90$; $\mathrm{p}=0.020$ ) and intra-stent residual stenosis [RR 1.04 (per \% unit increase); $95 \% \mathrm{Cl} 1.01-1.06 ; p=0.003]$. Conclusions: In this real world prospective registry, the cumulative incidence of stent thrombosis up to 5 year follow-up was

\footnotetext{
1 Hospital do Coração - Associação do Sanatório Sírio - São Paulo, SP. Correspondência: Professor Doutor J. Eduardo Sousa, Diretor Clínico. Rua Desembargador Eliseu Guilherme, 123 - Paraíso - São Paulo, SP - CEP 04004-030

E-mail: jesousa@uol.com.br

Recebido em: 14/5/2008 • Aceito em: 19/5/2008
} 
Costa RA, et al. Trombose de Stent Farmacológico no "Mundo-Real": Análise Crítica do Registro DESIRE (Drug-Eluting Stent in the Real World). Rev Bras Cardiol Invas. 2008;16(2):144-154.

complexa da lesão, múltiplos stents implantados por território miocárdico e subexpansão do stent.

DESCRITORES: Endoprótese, efeitos adversos. Angioplastia transluminal percutânea coronária. Trombose. Coronariopatia. Resultado de tratamento. rare $(1.6 \%)$, and associated to smoking, $\mathrm{PCI}$ in the $\mathrm{AMI}$ complex lesion morphology, multiple stents implanted per myocardial territory and subexpantion of the stent.

DESCRIPTORS: Stents, adverse effects. Angioplasty, transluminal, percutaneous coronary. Thrombosis. Coronary disease. Treatment outcome.

clínico prospectivo, não-randomizado, de braço único, com inclusão consecutiva de pacientes (inclusão em andamento), realizado em uma única instituição (Hospital do Coração - Associação do Sanatório Sírio - São Paulo, SP, Brasil). O estudo tem como objetivo investigar a evolução clínica tardia de pacientes tratados com SF. A partir de maio de 2002, a ICP com SF tem sido utilizada como estratégia preferencial nos pacientes encaminhados para tratamento percutâneo de rotina ou emergência na referida instituição. Foram incluídos pacientes com pelo menos uma lesão com estenose $\geq 50 \%$ e anatomia favorável para ICP. Pelo protocolo, não existem limitações no que se refere ao número de lesões-alvo e/ou vasos-alvo que podem ser tratados ou ao número de SF implantados. O estudo está em consonância com a Declaração de Helsinque no que se refere à investigação em humanos, e foi aprovado pelo Comitê de Ética da referida instituição.

\section{Procedimento}

As ICPs foram realizadas de acordo com as diretrizes atuais e a estratégia final do procedimento foi deixada a critério do operador. Os seguintes SF de primeira e segunda gerações foram utilizados: Cypher ${ }^{\circledR}$, liberador de sirolimus (Cordis Corporation, Warren, NJ, Estados Unidos); Taxus ${ }^{\circledR}$, liberador de paclitaxel (Boston Scientific, Natick, MA, Estados Unidos); Endeavor $^{\circledR}$, liberador de zotarolimus (Medtronic, Santa Rosa, CA, Estados Unidos); e Xience $\vee^{\circledR}$, liberador de everolimus (Abbott Vascular, Abbott Park, IL, Estados Unidos). Quando o resultado angiográfico imediato após o implante do stent demonstrava expansão subótima, era realizada pós-dilatação com cateter-balão de extensão mais curta que o stent, o qual era posicionado dentro das bordas do stent e insuflado a altas pressões (> 14 atm). Também eram permitidos implante de múltiplos stents e/ou tratamento de múltiplas lesões (por meio de procedimento estagiado ou não). Em pacientes com síndrome coronária aguda (SCA), estudo angiográfico e ICP (quando indicada) foram realizados em $<48$ horas da hospitalização. O protocolo antitrombótico consistia na administração de dois agentes antiplaquetários: ácido acetilsalicílico (AAS) e clopidogrel. O pré-tratamento (AAS 500 mg e clopidogrel 300-600 mg) foi realizado 24 horas antes da intervenção, para casos eletivos, ou de 3 a 6 horas antes do procedimento, para a SCA sem supradesnivelamento do segmento ST. No caso de ICP primária, a dose de iais do Registro DESIRE já foram reportados previamente ${ }^{7}$. Em resumo, o Registro DESIRE é um estudo 
ataque desses dois medicamentos foi administrada no momento do procedimento. Após a intervenção, a terapia com AAS era mantida indefinidamente, na dose de 100-200 mg/dia, e o clopidogrel, na dose de $75 \mathrm{mg}$ diários, era mantido inicialmente por 3 a 6 meses. A partir do final de 2006, seu uso foi estendido para 12 meses, de acordo com as novas recomendações do Food and Drug Administration (FDA) ${ }^{13}$. Durante o procedimento, foi administrada heparina endovenosa (70100 unidades $/ \mathrm{kg}$ ) para manter o tempo de coagulação ativado > 250 segundos (ou > 200 segundos no caso de ser administrado inibidor da glicoproteína IIb/IIla). Eletrocardiograma (ECG) com 12 derivações foi obtido de rotina antes do procedimento, imediatamente após o procedimento e depois de 24 horas. Exames laboratoriais de rotina incluíram enzimas cardíacas (CPK e CK-MB massa) pré-procedimento ( $<24$ horas), 1824 horas pós-procedimento, e diariamente até a alta hospitalar em caso de alteração das mesmas.

\section{Análise angiográfica}

A análise angiográfica qualitativa incluiu a avaliação do "território miocárdico" acometido, o qual foi definido como a porção do músculo cardíaco irrigada por uma artéria epicárdica maior (descendente anterior, circunflexa e coronária direita) e seus respectivos ramos. Em relação à análise quantitativa, após a administração intracoronária de nitrato $(50-200 \mu \mathrm{g})$, foram obtidos estudos angiográficos seriados, incluindo angiografia pré e pós-procedimento. As análises de angiografia coronária quantitativa (ACQ) foram realizadas por operadores experientes utilizando um sistema de análise quantitativa com detecção semi-automática das bordas luminais (QuantcorQCA-ACOM.PC versão 4.0 - Siemens, Munique, Alemanha). O diâmetro luminal mínimo (DLM) e o diâmetro de referência do vaso (DRV), obtido pela média dos segmentos 5-10 mm proximal e distal da lesão-alvo, foram utilizados para calcular o diâmetro de estenose (DE): DE $=(1-\mathrm{DLM} /$ DRV) x 100. Ganho imediato foi definido como a diferença do DLM pré e pós-procedimento (DLM pósprocedimento - DLM pré-procedimento).

\section{Objetivos, definições e seguimento clínico}

O objetivo primário do estudo foi a ocorrência de eventos cardíacos adversos maiores (ECAM) no seguimento clínico tardio (> 6 meses). Os ECAM incluíram: morte cardíaca, IAM e RLA. Como regra, todas as mortes foram consideradas cardíacas a não ser que uma causa não-cardíaca pudesse ser claramente estabelecida por estudo clínico e/ou patológico. O diagnóstico de IAM foi baseado no aparecimento de nova onda-Q patológica em $\geq 2$ derivações contíguas no ECG e/ou elevação da CK-MB massa > 3 vezes o limite superior da normalidade. A TS foi definida de acordo com as proposições do Academic Research Consortium (ARC): definitiva (presença de SCA com confirmação angiográfica ou anatomopatológica de oclusão do stent), provável (ocorrência de morte súbita < 30 dias pósprocedimento índice ou IAM no território miocárdico tratado, sem confirmação angiográfica da oclusão do stent) e possível (ocorrência de morte súbita > 30 dias após o procedimento índice). A TS ainda foi classificada de acordo com a ocorrência temporal: aguda $(\leq 24$ horas do procedimento), subaguda (ocorrência entre 24 horas e 30 dias), tardia (ocorrência entre 1 e 12 meses pós-procedimento) e muito tardia (ocorrência $>12$ meses pós-procedimento). O sucesso angiográfico foi definido como fluxo final TIMI 3, ausência de dissecções e estenose residual $<20 \%$ pela ACQ. O seguimento clínico foi realizado 1, 6 e 12 meses após o procedimento e anualmente até o quinto ano de acompanhamento, e consistiu de visita médica agendada ou contato telefônico, realizado segundo protocolo pré-definido. Todos os eventos adversos reportados, incluindo TS, foram independentemente adjudicados por um Comitê de Eventos Clínicos, incluindo três experientes profissionais da área de Cardiologia clínica e invasiva.

\section{Análise estatística}

As variáveis são apresentadas como freqüências ou médias \pm desvio padrão. A ocorrência de trombose em função do tempo foi descrita por uma curva de Kaplan-Meier. Para identificar fatores associados ao desenvolvimento de TS foram utilizados modelos de riscos proporcionais de Cox. Todas as variáveis foram inicialmente analisadas em análises bivariadas e, posteriormente, de forma múltipla. A permanência das variáveis no modelo múltiplo foi baseada no teste de razão de verossimilhanças. Os resultados dos modelos de Cox foram apresentados em razões de risco e respectivos intervalos de confiança de 95\%. O teste de interação entre alguns fatores do procedimento foi testado pelo teste de Mantel-Hantzel. Valor de $p<0,05$ foi considerado significativo. As análises foram realizadas com os programas estatísticos SPSS versão 16.0 (SPSS Inc., Chicago, IL, Estados Unidos) e STATA versão 10 (StataCorp LP, College Station, TX, Estados Unidos).

\section{RESULTADOS}

No total, 2.365 pacientes foram incluídos no Registro entre maio de 2002 e janeiro de 2008. A Figura 1 apresenta o fluxograma de inclusão dos pacientes. De maneira geral, os pacientes apresentaram perfil clínico de elevada complexidade (Tabela 1). A Tabela 2 apresenta os dados angiográficos. A artéria descendente anterior foi o vaso-alvo mais tratado $(35,8 \%)$, e considerando-se o total de lesões $(n=3.333$ ) 20,8\% delas eram localizadas na porção proximal desse vaso.

\section{Dados do procedimento}

No total, 5,2\% dos pacientes receberam inibidor da glicoproteína Ilb/IIla. As lesões foram pré-dilatadas em $50,7 \%$. A média do número de lesões e do número de 
Costa RA, et al. Trombose de Stent Farmacológico no "Mundo-Real": Análise Crítica do Registro DESIRE (Drug-Eluting Stent in the Real World). Rev Bras Cardiol Invas. 2008;16(2):144-154.

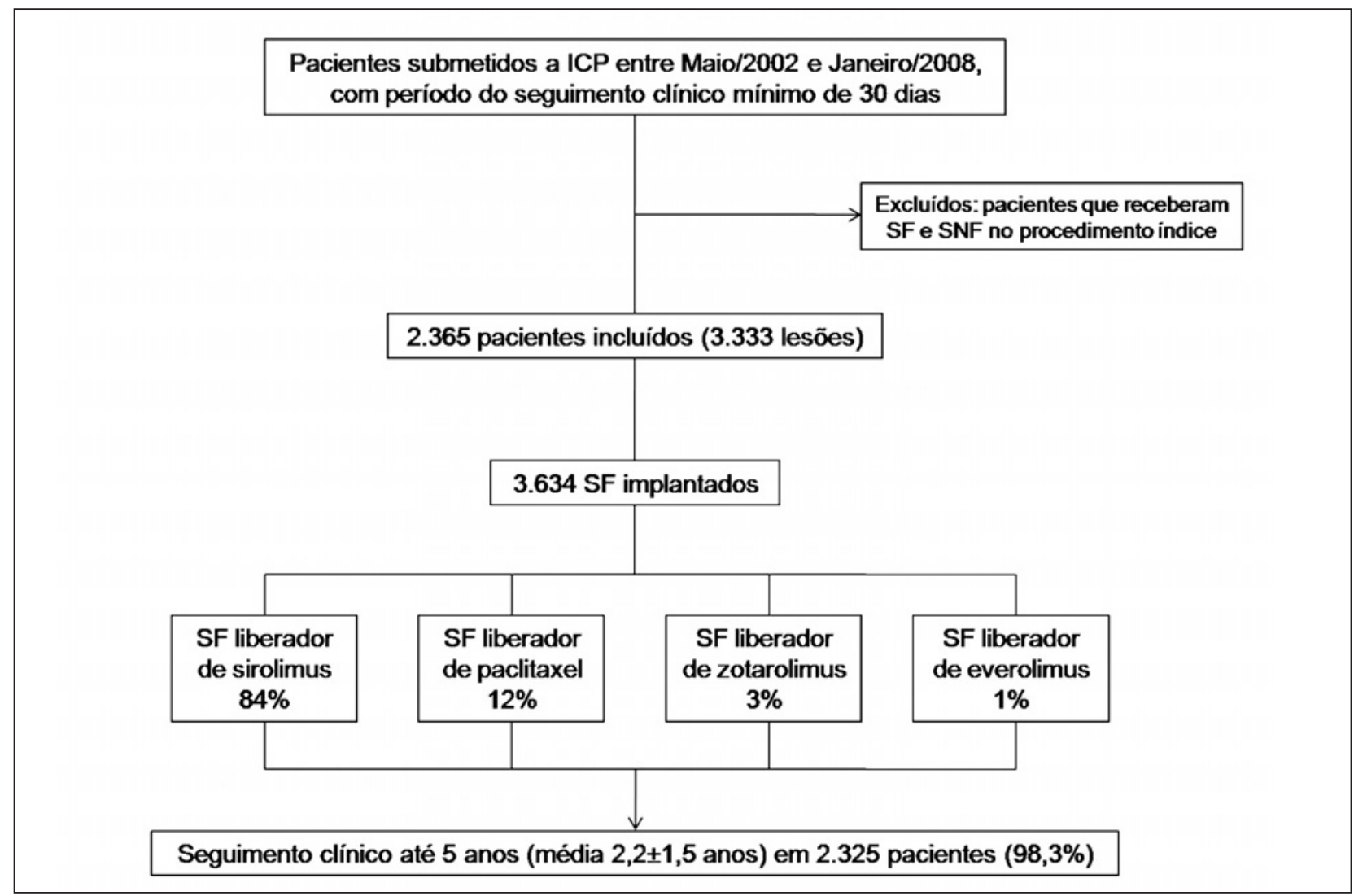

Figura 1 - Fluxograma de inclusão de pacientes no Registro DESIRE.

$\mathrm{ICP}=$ intervenção coronária percutânea; SF = stent farmacológico; SNF = stent não-farmacológico.

vasos tratados por paciente foi de 1,41 $\pm 0,65$, e 1,31 $\pm 0,54$, respectivamente. No total, 3.634 SF foram implantados (média de stents por paciente: 1,54 $\pm 0,76$ ) e $40 \%$ dos pacientes receberam múltiplos stents. A média da pressão de liberação do stent foi de $14,7 \pm 2,8 \mathrm{~atm}$. A pós-dilatação com cateter-balão foi aplicada em 43,7\% dos casos, e as médias de extensão, diâmetro e pressão de insuflação do balão foram de 12,13 \pm 4,11 mm, 3,28 $\pm 0,53 \mathrm{~mm}$, e 15,3 $\pm 3,9 \mathrm{~atm}$, respectivamente. Fluxo TIMI 3 pós-procedimento foi atingido em 99,6\% dos casos e o sucesso angiográfico foi de $98,6 \%$. Durante a fase hospitalar, todos os pacientes foram mantidos com terapia antiplaquetária dupla e a prescrição foi mantida na alta hospitalar de acordo com o protocolo. A Tabela 3 apresenta os resultados da ACQ.

\section{Eventos clínicos}

$\mathrm{Na}$ fase intra-hospitalar, morte cardíaca ocorreu em $0,3 \%$ dos pacientes e $2,1 \%$ evoluíram com IAM. No seguimento clínico tardio até cinco anos (média $2,2 \pm 1,5$ anos), as taxas cumulativas de ECAM foram de $10,6 \%$, incluindo morte cardíaca em 2,5\%, IAM em $4,5 \%$, e RLA em $3,6 \%$ dos pacientes. Durante o período de seguimento, $38(1,6 \%)$ pacientes evoluíram com TS (Figura 2). A maioria das tromboses foi classificada como definitiva, com confirmação angiográfica ( $n=23)$, e $42 \%$ ocorreram entre 1-12 meses de seguimento (Figura 3). As características individualizadas dos pacientes com TS são apresentadas na Tabela 4. Quando consideradas apenas as TS ocorridas no primeiro ano de evolução $(n=25)$, a média de tempo até o evento foi de 112,4 \pm 116,5 dias.

\section{Preditores de eventos adversos até cinco anos de seguimento clínico}

Os preditores independentes de ECAM foram: lesão-alvo em ponte de safena (razão de risco [RR] 1,63; intervalo de confiança [IC] 95\% 1,22-2,18; p=0,001), doença multiarterial (RR 1,39; IC 95\% 1,03-1,87; $p<0,001)$; estenose residual intra-stent ( $R R$ 1,3 [por aumento de unidade \%]; IC 95\% 1,10-1,50; $p=0,034)$, diabetes melito (RR 1,60; IC 95\% 1,10-2,20; $p=0,006$ ) e insuficiência renal (RR 1,50; IC 95\% 1,34-1,81; $p=0,004)$. A Tabela 5 apresenta a análise univariada para identificação de preditores de TS e os preditores independentes de TS identificados no modelo multivariado são apresentados na Tabela 6. Pelo teste de Mantel-Haenszel, evidenciou-se associação significativa entre a presença de calcificação moderada/grave e a realização de pós-dilatação para a ocorrência da TS $(p=0,007)$. Cerca de $20 \%$ dos pacientes com TS 
Costa RA, et al. Trombose de Stent Farmacológico no "Mundo-Real": Análise Crítica do Registro DESIRE (Drug-Eluting Stent in the Real World). Rev Bras Cardiol Invas. 2008;16(2):144-154.

apresentavam fração de ejeção (FE) do ventrículo esquerdo basal $<30 \%$ (versus $2,7 \%$ no restante da população; $p=0,003)$. Por último, as indicações de ICP consideradas mais complexas (off-label pelos critérios do FDA) tiveram maior prevalência entre pacientes que apresentaram TS $(81,6 \%)$ versus pacientes sem TS $(69,1 \%)$, no entanto sem significância estatística $(p=0,11)$.

\section{DISCUSSÃo}

Estudos prévios com pacientes provenientes do "mundo-real" demonstraram taxas de TS entre 1,3\% e $1,9 \%$ no seguimento tardio e fatalidade associada entre $39 \%$ e $45 \%{ }^{1,2}$. No Registro DESIRE, a TS ocorreu em $1,6 \%$ e a taxa de mortalidade foi de $47 \%$. Em nosso estudo, 16/38 pacientes apresentaram óbito como desfecho clínico inicial da TS. Dos 22/38 pacientes que apresentaram TS "definitiva" com comprovação angiográfica, 20 (90\%) foram submetidos a terapia de reperfusão, incluindo: $8 \mathrm{ICP}$ com balão, $5 \mathrm{ICP}$ com implante de SNF, 1 ICP com implante de SF, 1 cirurgia de revascularização do miocárdio e 2 terapias com trombolítico (3 pacientes foram submetidos a ICP, mas

TABELA 1

\section{Características clínicas basais}

\begin{tabular}{lc}
\hline Variável & $\mathbf{n}=\mathbf{2 . 3 6 5}$ \\
\hline Média das idades, anos & $63,9 \pm 11,5$ \\
Sexo masculino & $76,9 \%$ \\
Hipertensão & $75,7 \%$ \\
Diabetes melito & $28,6 \%$ \\
$\quad$ Em uso de insulina & $6,0 \%$ \\
Dislipidemia & $61,2 \%$ \\
Tabagismo atual & $32,1 \%$ \\
Histório familiar de DAC & $41,8 \%$ \\
IAM prévio & $22,2 \%$ \\
ICP prévia & $25,5 \%$ \\
CRM prévia & $26,7 \%$ \\
AVE prévio & $2,5 \%$ \\
Insuficiência renal* & $12,0 \%$ \\
Doença multiarterial & $59,9 \%$ \\
Doença vascular periférica & $4,4 \%$ \\
Apresentação clínica & \\
Angina estável & $27,6 \%$ \\
Angina instável & $26,0 \%$ \\
IAM & $15,4 \%$ \\
Isquemia silenciosa & $31,0 \%$ \\
\hline & \\
\hline
\end{tabular}

Valores são expressos como freqüência ou média \pm desvio padrão. * Definida como creatinina sérica basal $\geq 1,5 \mathrm{mg} / \mathrm{dL}$. AVE = acidente vascular encefálico; CRM = cirurgia de revascularização do miocárdio; DAC = doença arterial coronária; IAM = infarto agudo do miocárdio; ICP = intervenção coronária percutânea; $\mathrm{n}$ = número de pacientes. não obtiveram sucesso no cruzamento da lesão com o fio-guia). Entre os pacientes com terapia de reperfusão bem-sucedida ( $n=17), 77 \%(n=13)$ apresentaram-se assintomáticos ou com angina estável no seguimento clínico > 6 meses pós-TS, demonstrando que pacientes com TS documentada que são submetidos a terapia de reperfusão apresentam índices relativamente

TABELA 2

Características angiográficas

\begin{tabular}{lc}
\hline Variável & $\mathbf{n}=\mathbf{2 . 3 6 5}$ \\
\hline $\begin{array}{l}\text { Território miocárdico acometido* } \\
\text { (número de territórios = 2.931) }\end{array}$ \\
DA \\
Cx \\
CD \\
TCE \\
Características da lesão \\
(número de lesões = 3.333) \\
Ponte de veia safena \\
Localização ostial \\
Bifurcação \\
Angulação > 45 graus \\
Excentricidade \\
Calcificação moderada ou grave \\
Reestenose intra-stent \\
Lesão tipo B2 ou C (classificação ACC/AHA) \\
Fração de ejeção do ventrículo \\
esquerdo+< $40 \%$
\end{tabular}

Valores são expressos como freqüência ou média \pm desvio padrão. * O território miocárdico irrigado pela ponte de veia safena foi incluído nessa variável. + Dado disponível em apenas 28,2\% dos pacientes. $\mathrm{ACC}=$ American College of Cardiology; $\mathrm{AHA}=$ American Heart Association; $\mathrm{CD}=$ artéria coronária direita; $\mathrm{Cx}=$ artéria circunflexa; $\mathrm{DA}=$ artéria descendente anterior; $\mathrm{n}=$ número de pacientes; TCE = tronco de artéria coronária esquerda.

\section{TABELA 3}

Angiografia coronária quantitativa (número de lesões = 3.333)

\begin{tabular}{lcc} 
Variável quantitativa & $\begin{array}{c}\text { Pré- } \\
\text { procedimento }\end{array}$ & $\begin{array}{c}\text { Pós- } \\
\text { procedimento } \\
\text { (final)* }\end{array}$ \\
\hline Extensão da lesão & $16,42 \pm 8,17$ & - \\
DRV & $2,77 \pm 0,48$ & - \\
DLM & $1,03 \pm 1,74$ & $2,85 \pm 0,96$ \\
DE & $67,0 \pm 12,4$ & $4,8 \pm 3,6$ \\
Ganho imediato & - & $1,89 \pm 0,45$
\end{tabular}

Valores são expressos como média \pm desvio padrão. * Análise do segmento intra-stent. DE = diâmetro de estenose; DLM = diâmetro luminal mínimo; DRV = diâmetro de referência do vaso. 


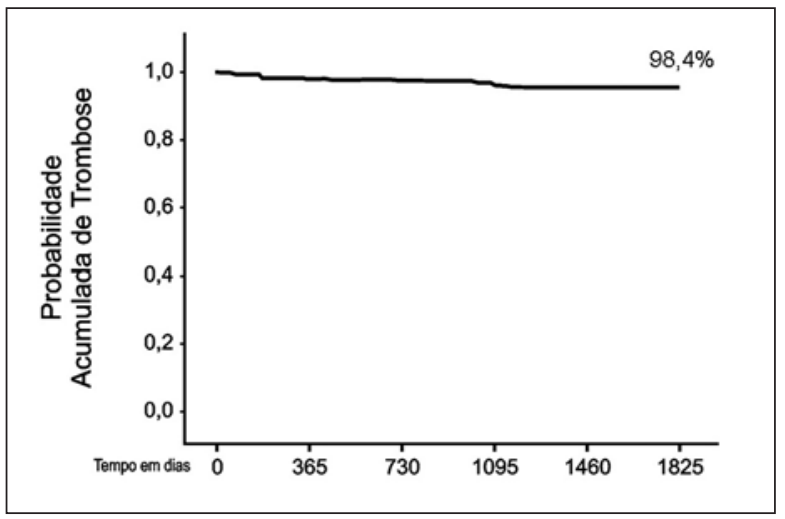

Figura 2 - Curva de sobrevida livre de trombose de stent até cinco anos de seguimento clínico.

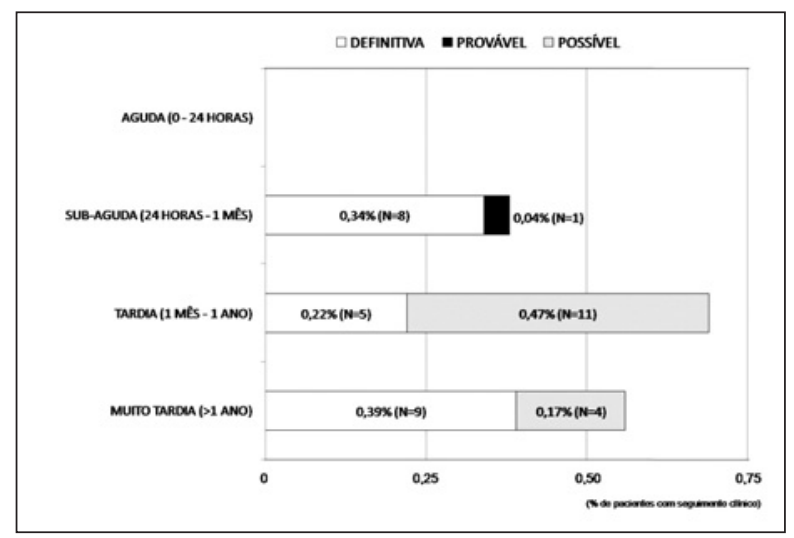

Figura 3 - Taxas de trombose de stent de acordo com as definições e a classificação temporal propostas pelo Academic Research Consortium.

altos de sucesso terapêutico e podem evoluir favoravelmente no seguimento tardio.

Uma série de estudos e metanálises conduzidos com metodologia adequada demonstrou, de maneira consistente, que os SF apresentam discreto aumento nas taxas de TS muito tardia quando comparados aos SNF em pacientes não-selecionados; no entanto, esse aumento da TS não se traduziu em aumento de óbito e IAM ${ }^{14-18}$. Isso pode ser explicado pela evolução clínica tardia "não-benigna" da reestenose com SNF em alguns casos. De fato, uma metanálise robusta recentemente apresentada, que incluiu grande número de pacientes estudados em séries randomizadas e nãorandomizadas (> $180 \mathrm{mil}$ ), demonstrou diminuição significativa de óbito e IAM com SF (Stone G, comunicação pessoal, American College of Cardiology, Chicago, Estados Unidos, 2008). No Registro DESIRE, a taxa total de TS até um ano de seguimento foi de 1,3\% ( $0,7 \%$ definitiva/provável), e pequeno aumento na ocorrência de TS foi observado > 1 ano; entretanto, esse incremento parece estar dentro das taxas projetadas para se manter benefício clínico superior ao dos SNF ${ }^{19}$.
Em pesquisa realizada por lakovou et al. ${ }^{1}$, que incluiu 2.229 pacientes do "mundo-real" tratados com SF, a taxa de TS aos 9 meses foi de 1,3\% (0,6\% até 30 dias e $0,7 \%$ entre $1-9$ meses) e os preditores independentes de TS foram: descontinuação prematura de terapia antiplaquetária, falência renal, lesão envolvendo bifurcação, diabetes e fração de ejeção do ventrículo esquerdo baixa. Em nossa análise, tanto o diabetes como a insuficiência renal foram preditores independentes de ECAM, mas não de TS. O estudo de lakovou et al. ${ }^{1}$ apresentou taxas relativamente mais altas de TS que o Registro DESIRE e a baixa incidência de eventos pode ter influenciado o impacto do diabetes na TS no Registro (análise univariada: RR 1,51 ; IC 95\% 0,78-2,91; $p=0,223)$. Em relação às bifurcações, a série de lakovou et al. ${ }^{1}$ incluiu $23 \%$ de bifurcações, sendo a maioria tratada com técnicas de dois stents. O tratamento de bifurcações com dois stents está associada a elevação das taxas de TS $^{20}$. No Registro DESIRE, apenas 3,8\% das lesões envolviam bifurcação, das quais $80 \%$ foram tratadas com apenas um stent (estratégia de stent provisional no ramo lateral). Mesmo assim, dois casos de TS em nossa série envolveram lesões em bifurcação tratadas com técnica de dois stents (Tabela 4). Adicionalmente, os pacientes com TS em nosso estudo apresentaram maior prevalência de disfunção ventricular grave $(\mathrm{FE}<30 \%)$ versus pacientes sem TS ( $p=0,003)$. Como esse dado, no entanto, foi reportado em $<30 \%$ da população estudada, essa variável não foi incluída na análise multivariada final.

Em nosso estudo, tabagismo, implante de múltiplos SF por território miocárdico e ICP no IAM foram identificados como preditores de TS. Uma metanálise dos estudos Taxus II, IV, V e VI também identificou o tabagismo e o implante de múltiplos stents como preditores independentes de trombose de $\mathrm{SF}^{21}$. Com relação à ICP na vigência de IAM, vários estudos recentes têm demonstrado associação significativa entre a ICP no IAM e a ocorrência desse evento ${ }^{12,22}$. Os motivos de tal associação ainda não estão completamente elucidados; no entanto, algumas explicações têm sido propostas, incluindo: lesão com atividade inflamatória contínua (não cessa com implante de SF), a qual pode apresentar maior potencial trombogênico; reendotelização tardia do SF; e desenvolvimento de aposição incompleta das hastes $(\mathrm{AIH})$ do stent em decorrência de lise posterior do trombo aderido à parede $^{23}$. A AIH é identificada pelo ultra-som intracoronário (USIC) e já foi descrita anteriormente ${ }^{24}$. Estudos de relatos de caso e análise histopatológica têm sugerido que a AIH pós-SF (principalmente a adquirida tardiamente) pode estar associada a trombose de SF muito tardia ${ }^{25,26}$. Infelizmente, o USIC não foi sistematicamente aplicado em nosso estudo, o que inviabilizou a análise da freqüência e do impacto clínico da AIH pós-SF em nossa população. Teoricamente, a AlH causada por lise do trombo poderia ser prevenida se o dimensionamento e a expansão do stent fossem maximizados de acordo com as referências do vaso. Nesse caso, o USIC pode ser muito útil. No entanto, 
TABELA 4

Características individuais dos pacientes que apresentaram evento clínico de trombose de stent no seguimento clínico

\begin{tabular}{|c|c|c|c|c|c|c|}
\hline Pac. & $\begin{array}{c}\text { Data do } \\
\text { procedimento } \\
\text { inicial }\end{array}$ & $\begin{array}{c}\text { Vaso(s) } \\
\text { tratado(s) }\end{array}$ & $\begin{array}{c}\text { Stent(s) } \\
\text { utilizado(s)* }\end{array}$ & $\begin{array}{c}\text { Tempo para } \\
\text { o evento }\end{array}$ & $\begin{array}{c}\text { Uso de } \\
\text { tienopiridínico } \\
\text { (prescrição) }\end{array}$ & $\begin{array}{l}\text { Evento } \\
\text { clínico }\end{array}$ \\
\hline 1 & $18 / 6 / 2002$ & DA & Cypher $^{\circledR}(1)$ & 8 dias & Sim (3 meses) & IAM sem supra ST \\
\hline 2 & $12 / 7 / 2002$ & DA & Cypher $^{\circledR}(1)$ & 12 dias & Não ${ }^{+}$(3 meses) & IAM \\
\hline 3 & 9/8/2002 & $\mathrm{Mg}$ & Cypher $^{\circledR}(2)$ & 405 dias & Não (3 meses) & Morte súbita \\
\hline 4 & 13/8/2002 & CD & Cypher ${ }^{\circledR}(3)$ & 432 dias & Não (3 meses) & Morte súbita \\
\hline 5 & $22 / 8 / 2002$ & $\mathrm{CD}$ & Cypher $^{\circledR}(1)$ & 205 dias & Não (3 meses) & Morte súbita \\
\hline 6 & 4/9/2002 & DA/Dg (bifurcação) & Cypher $^{\circledR}(2)$ & 1.160 dias & Não (6 meses) & IAM \\
\hline 7 & $5 / 9 / 2002$ & $\mathrm{DA}$ & Cypher $^{\circledR}(2)$ & 1.188 dias & Não (3 meses) & IAM \\
\hline 8 & $24 / 10 / 2002$ & DA & Cypher $^{\circledR}(1)$ & 299 dias & Não (3 meses) & Morte súbita \\
\hline 9 & $20 / 2 / 2003$ & DA & Cypher $^{\circledR}(1)$ & 79 dias & Sim (3 meses) & $\operatorname{IAM}(?)$ \\
\hline 10 & $31 / 3 / 2003$ & $\mathrm{Cx}$ & Cypher $^{\circledR}(1)$ & 189 dias & Não (3 meses) & Morte súbita \\
\hline 11 & 8/5/2003 & Cx e Mg & Cypher $^{\circledR}(2)$ & 470 dias & Não (6 meses) & Morte súbita \\
\hline 12 & $16 / 6 / 2003$ & DA & Cypher $^{\circledR}(1)$ & 1.202 dias & Não (3 meses) & IAM \\
\hline 13 & $1 / 7 / 2003$ & Dg & $\operatorname{Taxus}^{\circledR}(1)$ & 55 dias & Sim (6 meses) & IAM sem supra ST \\
\hline 14 & $5 / 8 / 2003$ & TCE & Cypher ${ }^{\circledR}(1)$ & 1.106 dias & Não (6 meses) & Morte súbita \\
\hline 15 & $25 / 9 / 2003$ & DA & Cypher $^{\circledR}(1)$ & 1.305 dias & Não (3 meses) & IAM \\
\hline 16 & 9/10/2003 & CD e Cx & Cypher $^{\circledR}(2)$ & 152 dias & Não ${ }^{\ddagger}$ (6 meses) & IAM \\
\hline 17 & $21 / 10 / 2003$ & DA & Cypher $^{\circledR}(1)$, Taxus $^{\circledR}(1)$ & 12 dias & Não ${ }^{\S}$ (12 meses) & IAM \\
\hline 18 & $21 / 10 / 2003$ & DA e Mg & Cypher $^{\circledR}(2)$ & 1.246 dias & Não (6 meses) & IAM \\
\hline 19 & 9/1/2004 & DA e CD & $\operatorname{Taxus}^{\circledR}(1), \operatorname{Taxus}^{\circledR}(2)$ & 331 dias & Sim (12 meses) & IAM \\
\hline 20 & $28 / 1 / 2004$ & CD e Cx & Cypher $^{\circledR}(1)$, Taxus $^{\circledR}(1)$ & 20 dias & Sim (12 meses) & IAM \\
\hline 21 & $26 / 2 / 2004$ & Cx & Cypher $^{\circledR}(1)$ & 171 dias & Não (3 meses) & PCR (IAM?) \\
\hline 22 & $2 / 3 / 2004$ & DA/Dg (bifurcação) & Cypher $^{\circledR}(2)$ & 116 dias & Sim (6 meses) & IAM \\
\hline 23 & $6 / 4 / 2004$ & Mg1 e Mg2 & Cypher $^{\circledR}(1)$, Taxus $^{\circledR}(1)$ & 338 dias & Não§ (12 meses) & Morte súbita \\
\hline 24 & $30 / 4 / 2004$ & Ponte de safena & Cypher $^{\circledR}(1)$ & 1.060 dias & Sim (sem aspirina) & IAM sem supra \\
\hline 25 & $14 / 4 / 2005$ & DA & Cypher $^{\circledR}(2)$ & 654 dias & Não (6 meses) & IAM \\
\hline 26 & 8/6/2005 & DA & $\operatorname{Taxus}^{\circledR}(2)$ & 61 dias & Sim (12 meses) & Morte súbita \\
\hline 27 & 3/8/2005 & $\mathrm{Cx}$ & Cypher $^{\circledR}(1)$ & 77 dias & Sim (3 meses) & Morte súbita \\
\hline 28 & $11 / 8 / 2005$ & DA & Taxus $^{\circledast}(1)$ & 473 dias & Não (6 meses) & IAM \\
\hline 29 & 6/9/2005 & DA e Mg & Cypher $^{\circledR}(2)$ & 760 dias & Não (6 meses) & IAM \\
\hline 30 & $12 / 12 / 2005$ & CD & Cypher $^{\circledR}(1)$ & 349 dias & Não (3 meses) & Morte súbita \\
\hline 31 & $10 / 4 / 2006$ & DA & Cypher $^{\circledR}(1)$ & 46 dias & Sim (3 meses) & Morte súbita \\
\hline 32 & $3 / 5 / 2006$ & DA e CD & Cypher $^{\circledR}(1)$, Cypher $^{\circledR}(2)$ & 73 dias & Sim (6 meses) & Morte súbita \\
\hline 33 & $29 / 5 / 2006$ & DA & $\operatorname{Taxus}^{\circledR}(1)$ & 3 dias & Não ${ }^{\S}$ (6 meses) & IAM \\
\hline 34 & $26 / 7 / 2006$ & CD e Cx & Cypher $^{\circledR}(2)$ & 11 dias & Sim (6 meses) & IAM \\
\hline 35 & $8 / 11 / 2006$ & Cxe Mg & Cypher $^{\circledR}(2)$, Cypher $^{\circledR}(1)$ & 6 dias & Sim (6 meses) & IAM \\
\hline 36 & $13 / 11 / 2006$ & Ponte de safena & Endeavor $^{\circledR}(1)$, Taxus $^{\circledR}(1)$ & 189 dias & Sim (12 meses) & IAM \\
\hline 37 & $13 / 11 / 2006$ & DA & Cypher $^{\circledR}(1)$ & 3 dias & Sim (sem aspirina) & Morte súbita \\
\hline 38 & 15/1/2008 & Dg e Mg & Cypher $^{\circledR}(1)$, Taxus $^{\circledast}(1)$ & 5 dias & Não§ (12 meses) & IAM sem supra \\
\hline
\end{tabular}

* Número entre parênteses indica número total de stent(s) implantado(s) no paciente e/ou vaso(s); ${ }^{+}$Interrompeu o uso de clopidogrel por causa de cirurgia vascular de urgência. ${ }^{\ddagger}$ Interrompeu o uso de clopidogrel e aspirina por causa de sangramento gastrointestinal. $\S$ Uso de clopidogrel interrompido sem motivo conhecido. ARC $=$ Academic Research Consortium; AVE $=$ acidente vascular encefálico; $\mathrm{CD}=$ artéria coronária direita; $\mathrm{CRM}=$ cirurgia de revascularização do miocárdio; $\mathrm{Cx}=$ artéria circunflexa; DA = artéria descendente anterior; Dg = ramo diagonal; IAM = infarto agudo do miocárdio; ICC = insuficiência cardíaca congestiva; ICP = intervenção coronária percutânea; $M g$ = ramo marginal; Pac. = paciente; PCR = parada cardiorrespiratória; $\mathrm{RCP}=$ ressuscitação cardiopulmonar; SF = stent farmacológico; SNF = stent não-farmacológico; $\mathrm{TCE}=$ tronco de artéria coronária esquerda. 
Continuação da TABELA 4

Características individuais dos pacientes que apresentaram evento clínico de trombose de stent no seguimento clínico

\begin{tabular}{|c|c|c|c|c|c|}
\hline Pac. & $\begin{array}{c}\text { Confirmação } \\
\text { angiográfica } \\
\text { (ou anatomopa- } \\
\text { tológica) }\end{array}$ & $\begin{array}{l}\text { Classificação da } \\
\text { trombose } \\
\text { (ARC) }\end{array}$ & Tratamento & $\begin{array}{c}\text { Desfecho na } \\
\text { fase intra-hospitalar }\end{array}$ & $\begin{array}{c}\text { Evolução clínica } \\
\text { tardia }\end{array}$ \\
\hline 1 & Sim & Definitiva & ICP com balão & Choque cardiogênico e óbito & - \\
\hline 2 & Sim & Definitiva & Trombólise & Sem complicações & Angina estável \\
\hline 3 & Não & Possível & - & - & - \\
\hline 4 & Não & Possível & - & - & - \\
\hline 5 & Não & Possível & - & - & - \\
\hline 6 & Sim & Definitiva & CRM & Sem complicações & Assintomático \\
\hline 7 & Sim & Definitiva & ICP sem sucesso & Sem complicações & Angina estável \\
\hline 8 & Não & Possível & - & - & - \\
\hline 9 & Não & Possível & - & Morte cardíaca & - \\
\hline 10 & Não & Possível & - & - & - \\
\hline 11 & Não & Possível & - & - & - \\
\hline 12 & Sim & Definitiva & ICP com SNF & Sem complicações & Assintomático \\
\hline 13 & Sim & Definitiva & Conservador & Sem complicações & Assintomático \\
\hline 14 & Não & Possível & - & - & - \\
\hline 15 & Sim & Definitiva & ICP com SNF & Sem complicações & Assintomático \\
\hline 16 & $\operatorname{SiM}(C x)$ & Definitiva & ICP com balão & Sem complicações & Angina estável \\
\hline 17 & Sim (Cypher, Taxus) & Definitiva & ICP com balão & Sem complicações & Morte súbita \\
\hline 18 & $\operatorname{Sim}(\mathrm{DA})$ & Definitiva & ICP com SF & Sem complicações & Assintomático \\
\hline 19 & $\operatorname{Sim}(\mathrm{DA})$ & Definitiva & ICP com balão & Sem complicações & Assintomático \\
\hline 20 & $\operatorname{Sim}(C D)$ & Definitiva & ICP com SNF & Sem complicações & Assintomático \\
\hline 21 & Não & Possível & RCP & Hipoxemia cerebral e óbito & - \\
\hline 22 & Sim & Definitiva & ICP sem sucesso & CRM, apresentou AVE & Morte não-cardíaca \\
\hline 23 & Não & Possível & - & - & - \\
\hline 24 & Sim & Definitiva & Conservador & Sem complicações & Assintomático \\
\hline 25 & Sim & Definitiva & ICP com SNF & Sem complicações & Assintomático \\
\hline 26 & Não & Possível & - & - & - \\
\hline 27 & Não & Possível & - & - & - \\
\hline 28 & Sim & Definitiva & ICP com balão & Sem complicações & Assintomático \\
\hline 29 & $\operatorname{Sim}(D A)$ & Definitiva & Trombólise & Sem complicações & Assintomático \\
\hline 30 & Não & Possível & - & - & - \\
\hline 31 & Não & Possível & - & - & - \\
\hline 32 & Não & Possível & - & - & - \\
\hline 33 & Sim & Definitiva & ICP com balão & Sem complicações & Assintomático \\
\hline 34 & $\operatorname{Sim}(C D, C x)$ & Definitiva & ICP com balão & Choque cardiogênico e óbito & - \\
\hline 35 & $\operatorname{Sim}(C x)$ & Definitiva & ICP com SNF & Sem complicações & ICC \\
\hline 36 & Sim (Endeavor) & Definitiva & ICP sem sucesso & Sem complicações & Assintomático \\
\hline 37 & Não & Provável & - & - & - \\
\hline 38 & $\operatorname{Sim}(D g)$ & Definitiva & ICP com balão & Sem complicações & Assintomático \\
\hline
\end{tabular}

* Número entre parênteses indica número total de stent(s) implantado(s) no paciente e/ou vaso(s); ${ }^{+}$Interrompeu o uso de clopidogrel por causa de cirurgia vascular de urgência. ${ }^{\ddagger}$ Interrompeu o uso de clopidogrel e aspirina por causa de sangramento gastrointestinal. $\S$ Uso de clopidogrel interrompido sem motivo conhecido. ARC = Academic Research Consortium; AVE $=$ acidente vascular encefálico; $\mathrm{CD}=$ artéria coronária direita; $\mathrm{CRM}=$ cirurgia de revascularização do miocárdio; $\mathrm{Cx}=$ artéria circunflexa; $\mathrm{DA}$ = artéria descendente anterior; $\mathrm{Dg}$ = ramo diagonal; IAM = infarto agudo do miocárdio; ICC = insuficiência cardíaca congestiva; $I C P=$ intervenção coronária percutânea; $M g$ = ramo marginal; $P$ ac. $=$ paciente; $P C R=$ parada cardiorrespiratória; $\mathrm{RCP}=$ ressuscitação cardiopulmonar; SF = stent farmacológico; SNF = stent não-farmacológico; TCE = tronco de artéria coronária esquerda. 
Costa RA, et al. Trombose de Stent Farmacológico no "Mundo-Real": Análise Crítica do Registro DESIRE (Drug-Eluting Stent in the Real World). Rev Bras Cardiol Invas. 2008;16(2):144-154.

TABELA 5

Análise univariada para preditores de trombose de stent

\begin{tabular}{lccc}
\hline Variável & Razão de risco & IC 95\% & Valor de p \\
\hline Dislipidemia & 0,52 & $0,28-0,99$ & 0,047 \\
Intervenção coronária percutânea no IAM & 4,08 & $1,56-10,66$ & 0,004 \\
Diabetes melito & 1,51 & $0,78-2,91$ & 0,223 \\
Calcificação moderada ou grave da lesão & 2,28 & $1,39-3,74$ & 0,001 \\
Angulação & 1,88 & $0,92-3,85$ & 0,083 \\
Morfologia excêntrica da lesão & 1,80 & $1,08-2,98$ & 0,024 \\
Múltiplos stents implantados por território miocárdico & 2,05 & $1,25-3,53$ & 0,005 \\
Pós-dilatação da lesão com cateter-balão & 0,63 & $0,36-1,08$ & 0,090 \\
Uso de stent Taxus & 1,63 & $0,89-3,02$ & 0,116 \\
Lesão tipo C (classificação ACC/AHA) & 2,34 & $1,18-4,64$ & 0,015 \\
Diâmetro do balão utilizado na pós-dilatação (por aumento de 1 mm) & 0,30 & $0,09-1,02$ & 0,054 \\
Extensão do balão utilizado na pós-dilatação (por aumento de 1 mm) & 1,08 & $1,00-1,18$ & 0,055 \\
Estenose residual intra-stent (por aumento de unidade \%) & 1,05 & $1,02-1,07$ & $<0,001$ \\
\hline ACC = American College of Cardiology; AHA = American Heart Association; IAM = infarto agudo do miocárdio; IC = intervalo de \\
confiança.
\end{tabular}

TABELA 6

Modelo multivariado demonstrando preditores independentes significativos de trombose de stent

\begin{tabular}{lccc}
\hline Variável & Razão de risco & IC 95\% & Valor de $\mathbf{p}$ \\
\hline Tabagismo atual & 2,59 & $1,18-5,67$ & 0,018 \\
Intervenção coronária percutânea no IAM & 3,50 & $1,31-9,40$ & 0,013 \\
Calcificação moderada ou grave da lesão & 2,38 & $1,34-4,23$ & 0,003 \\
Morfologia excêntrica da lesão & 1,86 & $1,03-3,34$ & 0,039 \\
Múltiplos stents implantados por território miocárdico & 1,81 & $1,09-3,02$ & 0,023 \\
Pós-dilatação da lesão com cateter-balão & 0,50 & $0,29-0,90$ & 0,020 \\
Estenose residual intra-stent (por aumento de unidade \%) & 1,04 & $1,01-1,06$ & 0,003 \\
\hline
\end{tabular}

IAM = infarto agudo do miocárdio; IC = intervalo de confiança.

o manejo clínico da $\mathrm{AlH}$ adquirida tardiamente ainda permanece controverso.

A expansão do stent também se mostrou um preditor de TS em nosso estudo ("estenose residual"). Mesmo uma sensível elevação no DE final (por unidade porcentual) demonstrou causar incremento na TS. Notavelmente, vários estudos já demonstraram que o DE final no segmento intra-stent é sistematicamente subestimado pela ACQ em comparação ao USIC ${ }^{27,28}$. Ou seja, lesões com resultado angiográfico considerado ótimo podem conter subexpansão substancial do stent. Tal achado aparenta ser mais proeminente em lesões que apresentam calcificação significativa (outro preditor de TS em nosso estudo) ${ }^{29}$. A subexpansão de stent é um importante preditor de falência de stent, incluindo
TS, e isso tem sido extensamente demonstrado tanto com SF como com SNF ${ }^{11,30}$. Um estudo com USIC e SF comparando lesões com TS versus lesões sem TS demonstrou que: 1) a área mínima do stent (AMS) era menor no grupo com trombose $\left(4,3 \pm 1,6 \mathrm{~mm}^{2}\right.$ versus $\left.\left.6,2 \pm 1,9 \mathrm{~mm}^{2} ; \mathrm{p}<0,001\right), 2\right)$ a expansão do stent era menor no grupo com trombose $(65 \pm 18 \%$ versus 85 $\pm 14 \% ; p<0,001), 3$ ) a presença de estenose residual no segmento de referência era mais freqüente no grupo com trombose $(67 \%$ versus $9 \%$; p < 0,001), e 4) os preditores independentes de TS eram subexpansão do stent $(p=0,03)$ e estenose residual do segmento de referência $(p=0,02)^{11}$. No Registro DESIRE, a seleção da extensão do stent objetivou a cobertura completa da lesão e a razão extensão do stent: extensão da lesão foi de 1,2. 
A realização de pós-dilatação com elevadas pressões de insuflação tem demonstrado ser eficaz na melhora da AMS e na otimização da expansão do stent $^{31}$. Em nosso estudo, $44 \%$ das lesões foram pósdilatadas, com média de pressão de insuflação $>15$ atm, e a realização de pós-dilatação foi um fator "protetor" contra a TS $(p=0,020)$. Logo, não foi surpresa a identificação de interação significativa entre calcificação e pós-dilatação para ocorrência de TS ( $p=0,007)$. Assim, considerando-se que as lesões com calcificação significativa estão freqüentemente associadas à subexpansão do stent e que a ACQ tende a subestimar a real "subexpansão" do stent, podemos especular que o implante de SF com realização de pós-dilatação (e mesmo guia de USIC) poderia aumentar a AMS, maximizar a expansão do stent e, potencialmente, meIhorar os resultados clínicos nesse subgrupo de elevado risco.

Finalmente, a interrupção da terapia antiplaquetária com tienopiridínico tem se mostrado um dos mais poderosos fatores preditores de TS pós-SF 1,2,12. Curiosamente, o uso de tienopiridínico não apresentou impacto significativo na TS em nossa análise. Uma possível explicação é o fato de que as informações coIhidas na fase inicial do estudo refletiram a utilização ou não da medicação prescrita em um determinado momento de seguimento clínico; dessa forma, a avaliação precisa do período de manutenção do uso de tienopiridínico (além do tempo estimado pela prescrição médica) (Tabela 4) e o tempo exato de sua interrupção não foram coletados em todos os pacientes para essa análise. Entre os que apresentaram TS nos primeiros 12 meses $(n=25), 12$ estavam em uso de clopidogrel, 5 interromperam o uso de acordo com o protocolo da época, 2 interromperam o uso por motivo de sangramento significativo, 2 estavam em monoterapia com clopidogrel (intolerância à aspirina), e 4 interromperam o uso de clopidogrel sem motivo conhecido.

\section{Limitações}

Este estudo representa a experiência clínica de uma instituição em que o SF tem sido utilizado como estratégia inicial em pacientes com indicação de ICP. Como o Registro DESIRE conta com inclusão contínua de pacientes por tempo indeterminado, a análise atual reflete os achados referentes a um período preestabelecido (maio de 2002 a janeiro de 2008). No período do estudo, foram excluídos os pacientes tratados com SNF ou com a combinação de SF e SNF. Por último, os diferentes tipos de SF foram implantados em proporções não-balanceadas, tendo sido as indicações baseadas em: 1) disponibilidade, 2) anatomia e localização da lesão, 3) quadro clínico, e 4) preferência do operador. Logo, o impacto de cada SF na ocorrência de TS não pode ser comparado de forma consistente.

\section{CONCLUSÕES}

Nesse Registro prospectivo de "mundo-real", a ocorrência de TS em pacientes complexos tratados com SF foi rara (incidência cumulativa de 1,6\% até 5 anos de seguimento); no entanto, metade dos pacientes que apresentaram tal evento teve desfecho clínico fatal. A maioria dos casos de trombose teve confirmação angiográfica de oclusão do stent e ocorreu entre 1 e 12 meses de seguimento clínico. Nessa análise, a TS foi significativamente associada com tabagismo, ICP no IAM, morfologia complexa da lesão, múltiplos stents implantados por território miocárdico e subexpansão do stent.

\section{AGRADECIMENTOS}

Os autores gostariam de reconhecer a dedicação, competência, e significativa colaboração dos seguintes profissionais envolvidos no Registro DESIRE: Dr. Cantídio Campos, Fábio Kawasetsu, Mariana T. Carballo, Valéria Ranieri, e Wagner Pinto.

\section{REFERÊNCIAS BIBLIOGRÁFICAS}

1. Iakovou I, Schmidt T, Bonizzoni E, Ge L, Sangiorgi GM, Stankovic G, et al. Incidence, predictors, and outcome of thrombosis after successful implantation of drug-eluting stents. JAMA. 2005;293(17):2126-30.

2. Airoldi F, Colombo A, Morici N, Latib A, Cosgrave J, Buellesfeld $L$, et al. Incidence and predictors of drug eluting stent thrombosis during and after discontinuation of thienopyridine treatment. Circulation. 2007;116(7):745-54.

3. Moses JW, Leon MB, Popma JJ, Fitzgerald PJ, Holmes DR, O'Shaughnessy C, et al. Sirolimus-eluting stents versus standard stents in patients with stenosis in a native coronary artery. N Engl J Med. 2003;349(14):1315-23.

4. Stone GW, Ellis SG, Cox DA, Hermiller J, O'Shaughnessy C, Mann JT, et al. A polymer-based, paclitaxel-eluting stent in patients with coronary artery disease. $\mathrm{N}$ Engl J Med. 2004;350(3):221-31.

5. Stone GW, Moses JW, Ellis SG, Schofer J, Dawkins KD, Morice MC, et al. Safety and efficacy of sirolimus- and paclitaxel-eluting coronary stents. N Engl J Med. 2007; 356(10):998-1008.

6. Lemos PA, Serruys PW, van Domburg RT, Saia F, Arampatzis CA, Hoye A, et al. Unrestricted utilization of sirolimuseluting stents compared with conventional bare stent implantation in the "real world": the Rapamycin-Eluting Stent Evaluated At Rotterdam Cardiology Hospital (RESEARCH) registry. Circulation. 2004;109(2):190-5.

7. Sousa AGMR, Costa Jr JR, Moreira A, Costa RA, Cano MN, Andrade GAM, et al. Evolução clínica tardia dos stents farmacológicos. segurança e eficácia até cinco anos do registro DESIRE. Rev Bras Cardiol Invas. 2007;15(3):221-7.

8. Carlsson J, von Wagenheim B, Linder R, Anwari TM, Qvist $\mathrm{J}$, Petersson I, et al. Is late stent thrombosis in drug-eluting stents a real clinical issue? A single-center experience and review of the literature. Clin Res Cardiol. 2007;96(2):86-93.

9. Lagerqvist B, James SK, Stenestrand U, Lindbäck J, Nilsson $\mathrm{T}$, Wallentin L. Long-term outcomes with drug-eluting stents versus bare-metal stents in Sweden. $N$ Engl J Med. 2007;356(10):1009-19. 
10. Melikian N, Wijns W. Drug-eluting stents: a critique. Heart. 2008;94(2):145-52.

11. Fujii K, Carlier SG, Mintz GS, Yang YM, Moussa I, Weisz $\mathrm{G}$, et al. Stent underexpansion and residual reference segment stenosis are related to stent thrombosis after sirolimuseluting stent implantation: an intravascular ultrasound study. J Am Coll Cardiol. 2005;45(7):995-8.

12. Park DW, Park SW, Park KH, Lee BK, Kim YH, Lee CW, et al. Frequency of and risk factors for stent thrombosis after drug-eluting stent implantation during long-term follow-up. Am J Cardiol. 2006;98(3):352-6.

13. Grines $\mathrm{CL}$, Bonow RO, Casey DE Jr, Gardner TJ, Lockhart PB, Moliterno DJ, et al. Prevention of premature discontinuation of dual antiplatelet therapy in patients with coronary artery stents: a science advisory from the American Heart Association, American College of Cardiology, Society for Cardiovascular Angiography and Interventions, American College of Surgeons, and American Dental Association, with representation from the American College of Physicians. Circulation. 2007;115(6):813-8.

14. Stone GW, Ellis SG, Colombo A, Dawkins KD, Grube E, Cutlip DE, et al. Offsetting impact of thrombosis and restenosis on the occurrence of death and myocardial infarction after paclitaxel-eluting and bare metal stent implantation. Circulation. 2007;115(22):2842-7.

15. Jensen LO, Maeng $M$, Kaltoft $A$, Thayssen $P$, Hansen $H H$, Bottcher $M$, et al. Stent thrombosis, myocardial infarction, and death after drug-eluting and bare-metal stent coronary interventions. J Am Coll Cardiol. 2007;50(5):463-70.

16. Stettler C, Wandel S, Allemann S, Kastrati A, Morice MC, Schömig A, et al. Outcomes associated with drug-eluting and bare-metal stents: a collaborative network meta-analysis. Lancet. 2007;370(9591):937-48.

17. Tu JV, Bowen J, Chiu M, Ko DT, Austin PC, He Y, et al. Effectiveness and safety of drug-eluting stents in Ontario. $N$ Engl J Med. 2007;357(14):1393-402.

18. Marzocchi A, Saia F, Piovaccari G, Manari A, Aurier E, Benassi A, et al. Long-term safety and efficacy of drugeluting stents: two-year results of the REAL (REgistro AngiopLastiche dell'Emilia Romagna) multicenter registry. Circulation. 2007;115(25):3181-8.

19. Garg P, Cohen DJ, Gaziano T, Mauri L. Balancing the risks of restenosis and stent thrombosis in bare-metal versus drug-eluting stents: results of a decision analytic model. J Am Coll Cardiol. 2008;51(19):1844-53.
20. Costa RA, Moussa ID. Percutaneous treatment of coronary bifurcation lesions in the era of drug-eluting stents. Minerva Cardioangiol. 2006;54(5):577-89.

21. Ellis SG, Colombo A, Grube E, Popma J, Koglin J, Dawkins $\mathrm{KD}$, et al. Incidence, timing, and correlates of stent thrombosis with the polymeric paclitaxel drug-eluting stent: a TAXUS II, IV, V, and VI meta-analysis of 3,445 patients followed for up to 3 years. J Am Coll Cardiol. 2007;49 (10):1043-51.

22. Daemen J, Wenaweser P, Tsuchida K, Abrecht L, Vaina S, Morger C, et al. Early and late coronary stent thrombosis of sirolimus-eluting and paclitaxel-eluting stents in routine clinical practice: data from a large two-institutional cohort study. Lancet. 2007;369(9562):667-78.

23. Sianos G, Papafaklis MI, Daemen J, Vaina S, van Mieghem CA, van Domburg RT, et al. Angiographic stent thrombosis after routine use of drug-eluting stents in ST-segment elevation myocardial infarction: the importance of thrombus burden. J Am Coll Cardiol. 2007;50(7):573-83.

24. Mintz GS, Weissman NJ. Intravascular ultrasound in the drugeluting stent era. J Am Coll Cardiol. 2006;48(3):421-9.

25. Feres F, Costa JR Jr, Abizaid A. Very late thrombosis after drugeluting stents. Catheter Cardiovasc Interv. 2006;68(1):83-8.

26. Virmani R, Farb A, Guagliumi G, Kolodgie FD. Drug-eluting stents: caution and concerns for long-term outcome. Coron Artery Dis. 2004;15(6):313-8.

27. Costa Jr JR, Mintz GS, Carlier SG, Fujii K, Sano K, Kimura $M$, et al. Intravascular ultrasound assessment of drug-eluting stent expansion. Am Heart J. 2007;153(2):297-303.

28. Costa RA, Mintz GS, Carlier SG, Lansky AJ, Moussa I, Fujii $\mathrm{K}$, et al. Bifurcation coronary lesions treated with the "crush" technique: an intravascular ultrasound analysis. J Am Coll Cardiol. 2005;46(4):599-605.

29. Cook S, Wenaweser P, Togni M, Billinger M, Morger C, Seiler $C$, et al. Incomplete stent apposition and very late stent thrombosis after drug-eluting stent implantation. Circulation. 2007;115(18):2426-34.

30. Takebayashi H, Kobayashi Y, Mintz GS, Carlier SG, Fujii K, Yasuda $\mathrm{T}$, et al. Intravascular ultrasound assessment of lesions with target vessel failure after sirolimus-eluting stent implantation. Am J Cardiol. 2005;95(4):498-502.

31. Brodie BR. Adjunctive balloon postdilatation after stent deployment: is it still necessary with drug-eluting stents? J Interv Cardiol. 2006;19(1):43-50. 